\title{
Modelling studies on the investigation of non-linear optical properties of some $\mathbf{E x}^{\mathrm{n}} \mathrm{Box}$ cyclophanes
}

\author{
Koray SAYIN ${ }^{1,2 *}$ iD, Majid REZAEIVALA ${ }^{3}$ \\ ${ }^{1}$ Sivas Cumhuriyet University, Faculty of Science, Department of Chemistry 58140 Sivas, Turkey \\ ${ }^{2}$ Sivas Cumhuriyet University, Advanced Research and Application Center (CUTAM) 58140 Sivas, Turkey \\ ${ }^{3}$ Hamedan University of Technology, Department of Chemical Engineering, Hamedan 65155, Iran
}

\begin{abstract}
Modelling studies on some $\mathrm{Ex}^{\mathrm{n}}$ Box Cyclophanes were performed at HF/6-31G(d) level. Structural properties are examined in detail. Density of state (DOS) spectrum and molecular orbital energy diagram of related compounds at ground state were calculated and contour plots of significant molecular orbitals were investigated in detail. Some quantum chemical descriptors were calculated in the gas phase to examine the non-linear optical properties. Finally, UV-VIS spectrum of the mentioned compounds was calculated and examined in gas phase, toluene, chloroform, ethanol, methanol, 1,2-ethanediol, water and n-methylformamidemixture. As a result, $\mathrm{EX}^{2.2} \mathrm{Box}^{4+}$ was found as the best candidate to NLO applications.
\end{abstract}

\section{Article info \\ History:}

Received:11.02.2020

Accepted:06.05.2020

Keywords:

Ex $^{\mathrm{n}}$ Box,

Spectral analyses,

Modeling studies,

Ab-initio,

Optical property.

\section{Introduction}

Referred [1] to as $\mathrm{Ex}^{\mathrm{n} B o x^{4+}}$ compounds, tetracationic cyclophanes is produced by reacting rigid bipyridylbased linkers, in which $\mathrm{n}$ is the number of $\mathrm{p}$-phenylene spacers having been employed toward various chemistry [2,3]. The recent advent of extended viologen boxes, $\mathrm{EX}^{\mathrm{n}} \mathrm{Box}^{4+}$ has given rise to the opportunity to construct mechanomolecules out of ever larger recognition units due to the longer dimensions of their cavities. EXBox ${ }^{4+}$ has an extraordinary ability to recognize polycyclic aromatic hydrocarbons (PAHs). Even larger EX $\mathrm{X}^{\mathrm{n}}$ Boxes possessing viologen extensions are likely to construct, such as ptertphenylene-hexamethylated for the sake of solubility, dramatically reducing its affinity for guestsas well as bis-p-phenylene-ethylene spacers, resulting in high-yielding preparation of the corresponding host, $\mathrm{EX}^{2.2} \mathrm{Box}^{4+}$. Tetracationic cyclophanes are often used due to the favorable electronic, optizal and redox properties and diverse binding ability. These macrocyclic compounds generrally possess a two $\pi$ deficient cavity on the extended planes and two aromatic linkers [4]. These compounds have been used in the development of axially chiral system, liquid crystal and nonlinear optical properties [5,6]. The cyclophanes also exhibit optical properties that are influenced by a nonconventional internal charge transfer process, stemming from the strained cyclobutane core. In this project, the attempt is made to investigate $\mathrm{CBPQT}^{4+}\left(\mathrm{BlueBox}^{4+}\right), \mathrm{EXBox}^{4+}$, $\mathrm{EX}^{2} \mathrm{Box}^{4+}, \mathrm{EX}^{0.8} \mathrm{Box}^{4+}$ and $\mathrm{EX}^{2.2} \mathrm{Box}^{4+}$ as computationaly tecniques were those who synthesized BlueBox ${ }^{4+}, \mathrm{EXBox}^{4+}, \mathrm{EX}^{2} \mathrm{Box}^{4+}, \mathrm{EX}^{0.8} \mathrm{Box}^{4+}$ and $\mathrm{EX}^{2.2} \mathrm{Box}^{4+}$, respectively [7-10]. Schematic structures of these compounds are displayed in Scheme 1.

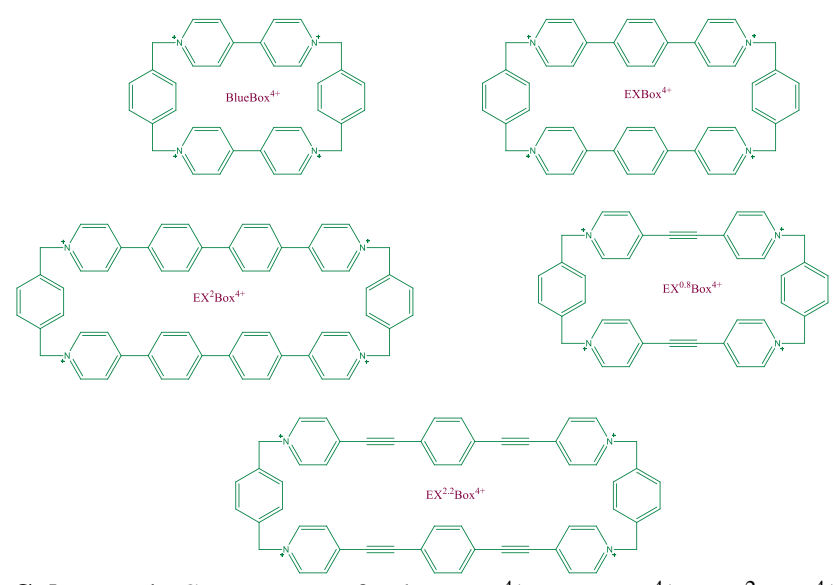

Scheme 1. Structures of BlueBox ${ }^{4+}, \mathrm{EXBox}^{4+}, \mathrm{EX}^{2} \mathrm{Box}^{4+}$, $\mathrm{EX}^{0.8} \mathrm{Box}^{4+}$ and $\mathrm{EX}^{2.2} \mathrm{Box}^{4+}$. 
Quantum chemical analyses of these structures are completed at HF/6-31G(d) level in vacuum. Initially, these structures are optimized at the same level of theory. To investigate the non-linear optical (NLO) properties, their molecular orbital energy diagrams are plotted by selecting the degeneracy threshold as 0.01 a.u. Significant molecular orbitals are determined and their contour diagrams are calculated and examined in detail. Density of state (DOS) spectrum of related compounds is calculated and examined. UV-VIS spectrum of mentioned molecules are calculated at the same level of theory in each area and the wavelength of main band is examined. Finally, some quantum chemical descriptors are calculated in gas phase, toluene, chloroform, ethanol, methanol, 1,2ethanediol, water and n-methylformamide-mixture.

\section{Methods}

Theoretical calculations of some Ex ${ }^{\mathrm{n}}$ Box Cyclophanes were performed at HF method with 6-31G(d) basis set in vacuum. Gaussian 5.0.8 [11], Gaussian09 AS64LG09RevD.01 [12], Chem-Bio Office [13] and GaussSum [14] were used in the whole analyses and structural properties of $\mathrm{BlueBox}^{4+}, \mathrm{EXBox}^{4+}$, $\mathrm{EX}^{2} \mathrm{Box}^{4+}, \mathrm{EX}^{0.8} \mathrm{Box}^{4+}$ and $\mathrm{EX}^{2.2} \mathrm{Box}^{4+}$ were examined. Ultraviolet-Visible (UV-VIS) spectrum of mentioned molecules was calculated in different eight phases which are gas phase $(\varepsilon=1)$, toluene $(\varepsilon=2.3741)$, chloroform $(\varepsilon=4.7113)$, ethanol $(\varepsilon=24.852)$, methanol $(\varepsilon=32.613), \quad 1,2$-ethanediol $\quad(\varepsilon=40.245), \quad$ water $(\varepsilon=78.3553)$ and n-methylformamide-mixture ( $\varepsilon=181.56)$. In these calculations, the time-dependent (TD) method was taken into consideration. As for the solvent model, CPCM method was used in solvent calculations. In the determination of NLO properties, urea was selected as a reference material. Some quantum chemical descriptors (QCDs) were used to determine the NLO activity and calculated by using Eq. (1) - (15):

$$
\begin{aligned}
& I=-E_{\text {HOMO }} \\
& A=-E_{L U M O} \\
& E_{G A P}=E_{L U M O}-E_{H O M O} \\
& \eta=\frac{I-A}{2}=\frac{E_{L U M O}-E_{H O M O}}{2} \\
& \sigma=\frac{1}{\eta} \\
& \sigma_{O}=\frac{1}{E_{G A P}}
\end{aligned}
$$

$$
\begin{aligned}
& \chi=\frac{|I+A|}{2}=\frac{\left|-E_{\text {номо }}-E_{\text {LUMO }}\right|}{2} \\
& C P=-\chi \\
& \Delta N_{\text {Max }}=-\frac{C P}{\eta} \\
& \omega=\frac{C P^{2}}{2 \eta} \\
& N=\frac{1}{\omega} \\
& \mu=\sqrt{\mu_{x}^{2}+\mu_{y}^{2}+\mu_{z}^{2}} \\
& \alpha=\frac{1}{3}\left(\alpha_{x x}+\alpha_{y y}+\alpha_{z z}\right) \\
& \Delta a=\frac{1}{\sqrt{2}}\left[\left(a_{x x}-a_{y y}\right)^{2}+\left(a_{y y}-a_{z z}\right)^{2}+\left(a_{z z}-a_{x x}\right)^{2}+\right. \\
& \left.6 a_{x z}{ }^{2}+6 a_{x y}{ }^{2}+6 a_{y z}{ }^{2}\right]^{1 / 2} \\
& \beta=\left[\left(\beta_{x x x}+\beta_{x y y}+\beta_{x z z}\right)^{2}+\left(\beta_{y y y}+\beta_{x y}+\beta_{y z z}\right)^{2}\right. \\
& \left.+\left(\beta_{z z z}+\beta_{x z z}+\beta_{y z z}\right)^{2}\right]^{1 / 2}
\end{aligned}
$$

where Koopmans theorem was taken into consideration in the obtaining of the ionization energy and electron affinities [15]. Calculated in each solvent for studied compounds were related quantum chemical parameters.

\section{Results and Discussion}

\subsection{Molecular structure}

BlueBox ${ }^{4+}, \mathrm{EXBox}^{4+}, \mathrm{EX}^{2} \mathrm{Box}^{4+}, \mathrm{EX}^{0.8} \mathrm{Box}^{4+}$ and $\mathrm{EX}^{2.2} \mathrm{Box}^{4+}$ are optimized at $\mathrm{HF} / 6-31 \mathrm{G}(\mathrm{d})$ level in vacuum. Optimized structures of mentioned $\mathrm{Ex}^{\mathrm{n} B}$ Boxes are represented in Fig. 1.

Given the optimized structure of studied molecules, the whole compounds are quite tense. There are a lot of $\pi$ electrons. Molecular planarity is distorted in each compound. Especially, benzene rings on both sides are nearly perpendicular to other benzene rings. IR spectrum of each studied Ex Boxes was calculated and no imaginary frequency was observed in the results. Bond lengths, represented in BlueBox ${ }^{4+}$ are calculated as $111^{\circ}, 112^{\circ}, 116^{\circ}, 116^{\circ}$ and $115^{\circ}$ for BlueBox ${ }^{4+}$, $\mathrm{EXBox}^{4+}, \mathrm{EX}^{2} \mathrm{Box}^{4+}, \mathrm{EX}^{0.8} \mathrm{Box}^{4+}$ and $\mathrm{EX}^{2.2} \mathrm{Box}^{4+}$, respectively. These angles imply the structural tension. 

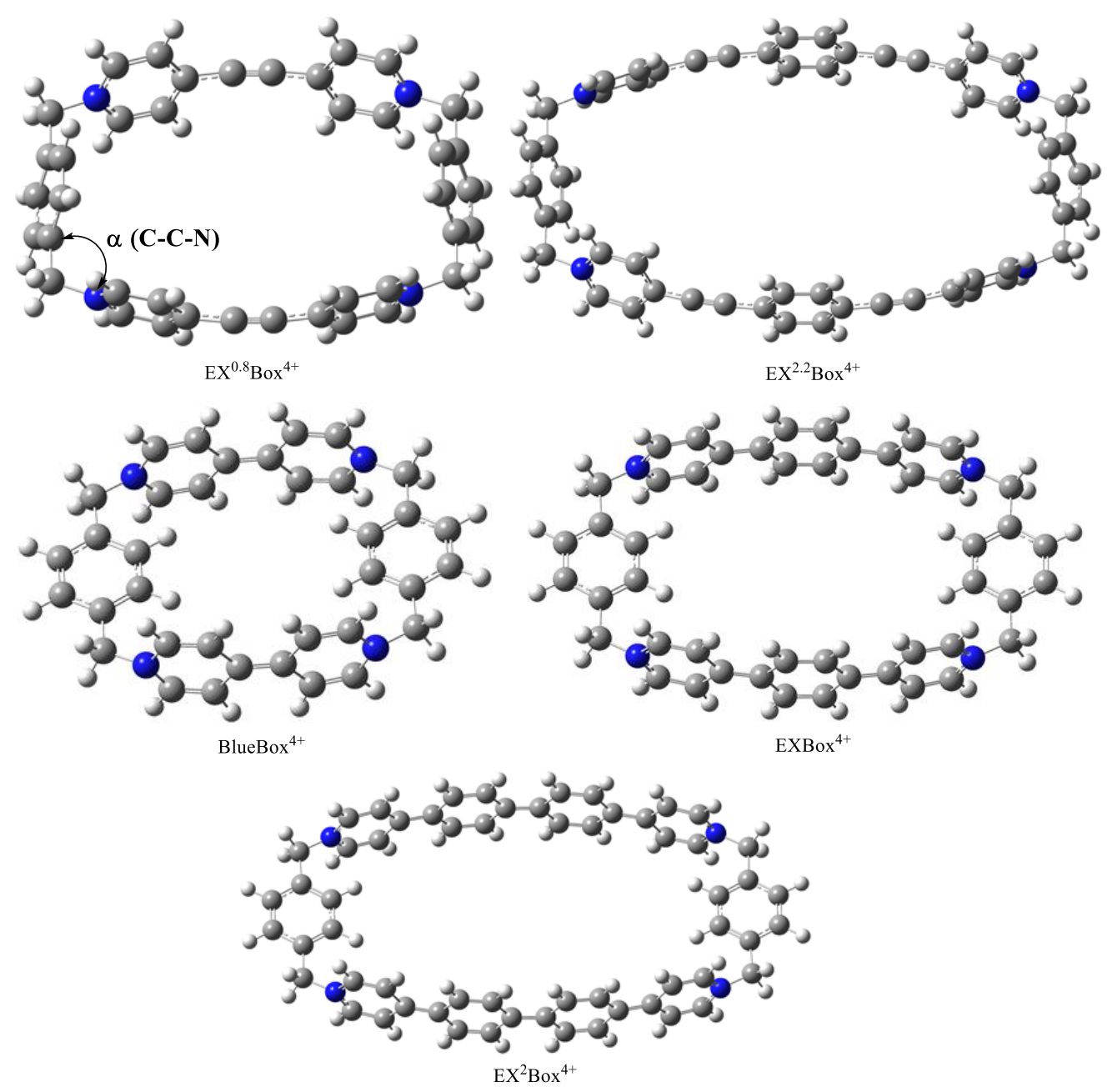

Fig. 1. Ground state structure of studied of some $\mathrm{Ex}^{\mathrm{n}} \mathrm{B}$ ox Cyclophanes.

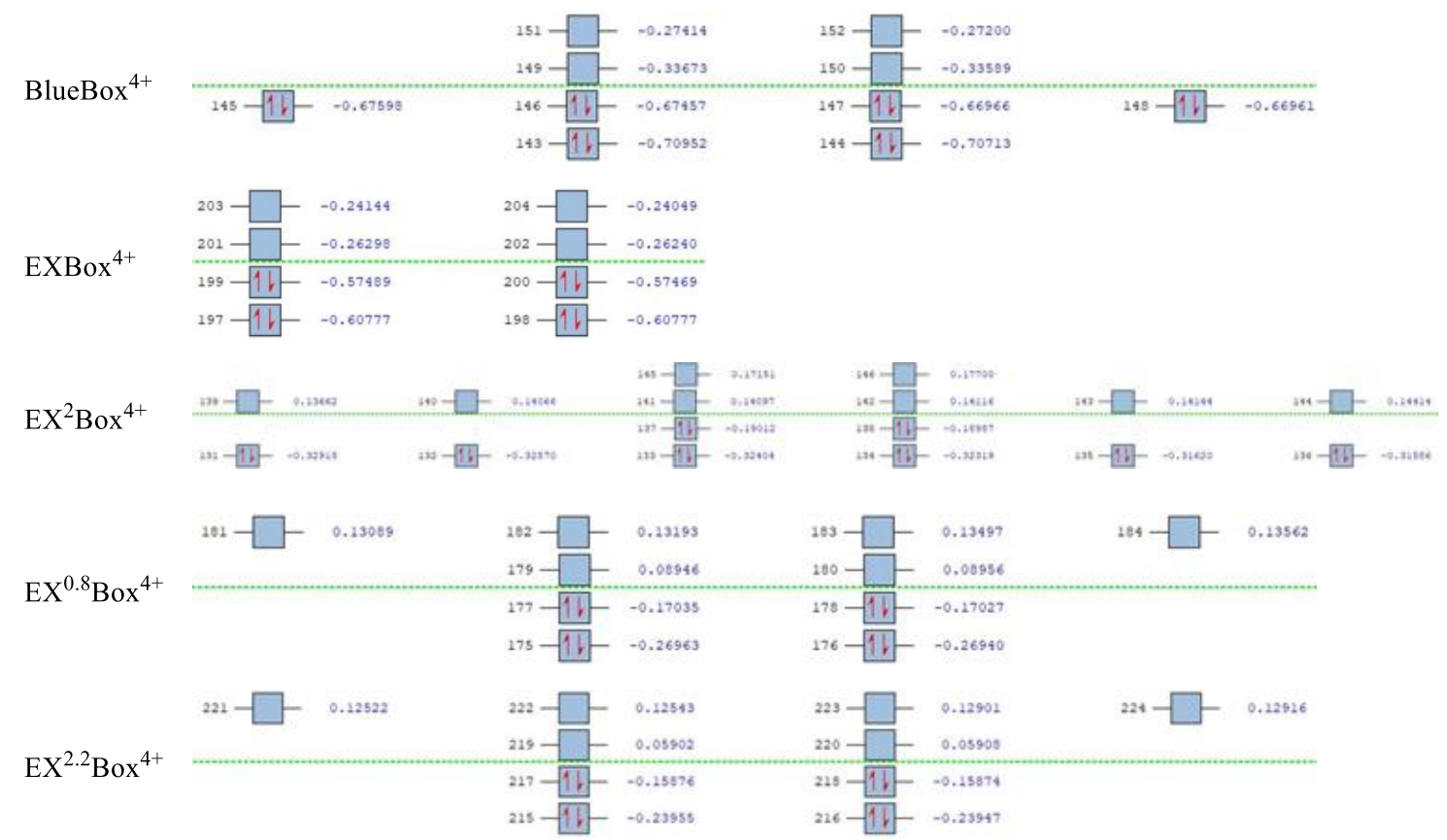

Fig. 2. The molecular orbital energy diagram of mentioned compounds. 


\subsection{Molecular orbital energy diagram (MOED), contour diagram and density of state (DOS) spectrum}

Energy diagram of molecular orbitals (MOEDs) are important to detect the electron fluidity and indirectly to understand the optical property. The lower energy gap means the more electron mobility. Electrons can transfer from ground state to excited state. However, the determination of related molecular orbitals is important. In this stage, MOEDs are calculated by defining the degeneracy threshold as 0.01 a.u, represented in Fig. 2.

Frontier molecular orbitals, the highest occupied molecular orbital (HOMO) and the lowest unoccupied molecular orbitals are important in the determination of electronic properties of compounds. In addition to these molecular orbitals, degenerate molecular orbitals are important in the determination of electronic properties of chemicals, too. Significant molecular orbitals can be seen easily from Fig. 2. For instance, LUMO+1 (150. MO), LUMO (149. MO), HOMO (148. MO), HOMO-1 (147. MO), HOMO-2 (146. MO) and HOMO-3 (145. MO) are important for BlueBox ${ }^{4+}$. Contour plots of selected molecular orbitals in BlueBox ${ }^{4+}$ are showed in Fig. 3 and selected molecular orbitals in each box are represented in Supplemental Material.

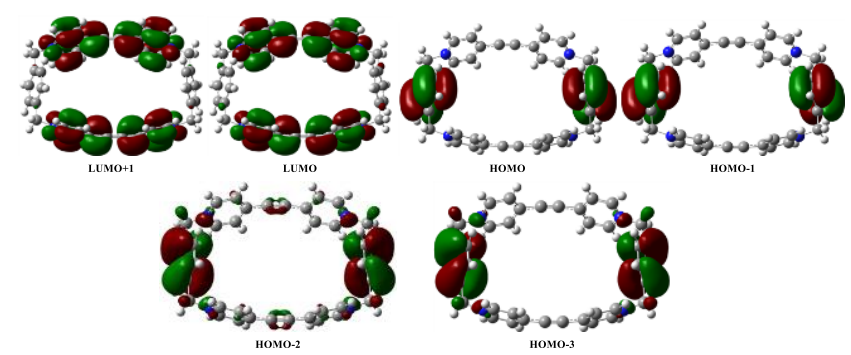

Fig. 3. Contour diagrams of selected MOs in BlueBox ${ }^{4+}$.

According to Fig. 2 and Fig. 3, there are red and green loops in contour plots. In occupied molecular orbitals, these loops imply the electron delocalization region. As for the unoccupied molecular orbital, if related compound accepts electron(s), this electron will be delocalized on mentioned loops. There are two electrons in HOMO and these electrons are delocalized on benzene rings where both side on molecule structure. In the determination of optical properties, contour plots of occupied molecular orbitals are more important than those of unoccupied molecular orbitals. Because the optical property is directly proportional to electron mobility. The more the electron mobility, the greater the optical property is expected. Additionally, the electron mobility is expected to be on the entire molecular surface for the increase of optical property. In BlueBox ${ }^{4+}$, electron mobility occurs only both sides on molecule and it is negative advantage for optical properties. As for the other compounds, electrons in occupied molecular orbital are generally delocalized on the entire molecular surface. Therefore, it is supposed that optical properties of $\mathrm{EXBox}^{4+}$, $\mathrm{EX}^{2} \mathrm{Box}^{4+}, \mathrm{EX}^{0.8} \mathrm{Box}^{4+}$ and $\mathrm{EX}^{2.2} \mathrm{Box}^{4+}$ are better than that of BlueBox ${ }^{4+}$. DOS spectrum is calculated for studied Ex ${ }^{n}$ Boxes as represented in Fig. 4.
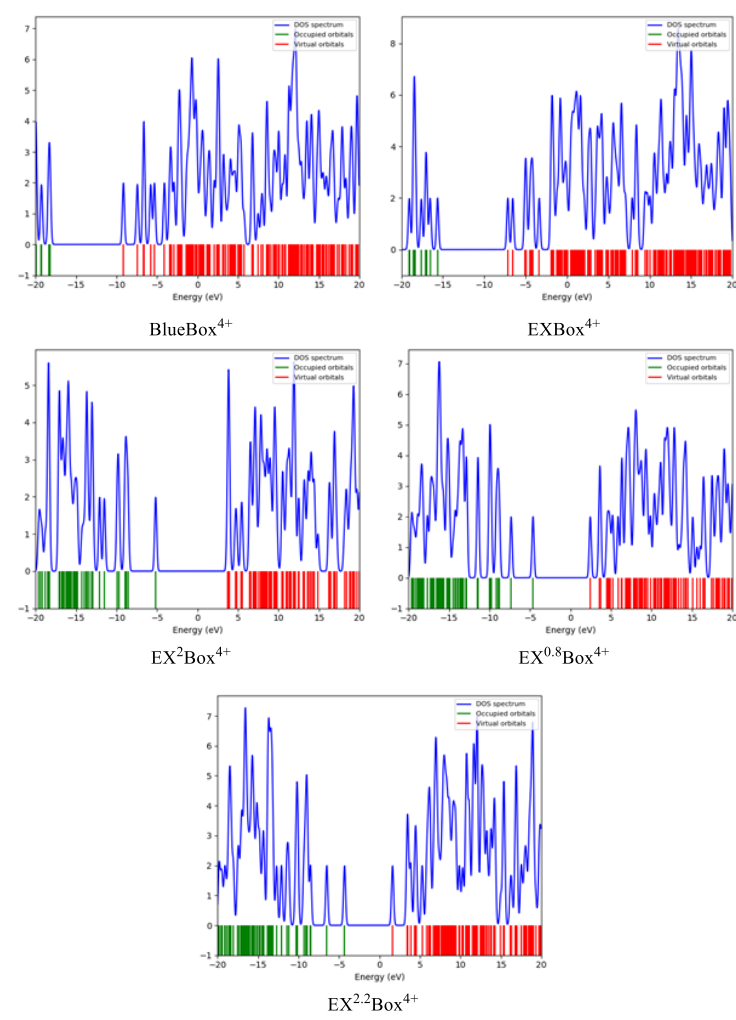

Fig. 4. DOS spectrum of studied of $\mathrm{Ex}^{\mathrm{n}} \mathrm{B}$ ox Cyclophanes

According to Fig. 4, molecular orbitals in the range of $(-20.0)-(+20.0) \mathrm{eV}$ are taken into consideration. While green peaks represent the occupied molecular orbitals, red peaks represent the unoccupied molecular orbitals. The more peak number in occupied and unoccupied molecular orbitals means that electrons can be easily transferred from ground state to excited state. In this range, there no more occupied molecular orbitals in BlueBox ${ }^{4+}$ and EXBox ${ }^{4+}$. Therefore, it is expected that optical properties of $\mathrm{EX}^{2} \mathrm{Box}^{4+}$, $\mathrm{EX}^{0.8} \mathrm{Box}^{4+}$ and $\mathrm{EX}^{2.2} \mathrm{Box}^{4+}$ outperforms than the others.

\subsection{Simulated UV-Vis spectrum}

UV-VIS spectrum gives significant results about conjugation degree, aromaticity and electronic 
transitions. A band in the UV-VIS spectrum consists of many electronic transitions. A lot of bands or shoulder in UV-VIS spectrum means a lot of electronic transitions. Additionally, bigger wavelength value means the lower energy to electronic transitions. UV-
VIS spectrum of mentioned $\mathrm{Ex}^{\mathrm{n}}$ Boxes is calculated in gas phase, toluene, chloroform, ethanol, methanol, 1,2ethanediol, water and n-methylformamide-mixture. The wavelengths of main bands are given in Table 1 .

Table 1. Calculated wavelength $(\mathrm{nm})$ of main band of studied $\mathrm{Ex}^{\mathrm{n}}$ Boxes in each solvent area

\begin{tabular}{llllll}
\hline Area & BlueBox $^{4+}$ & $\mathrm{EXBox}^{4+}$ & $\mathrm{EX}^{2} \mathrm{Box}^{4+}$ & $\mathrm{EX}^{0.8} \mathrm{Box}^{4+}$ & $\mathrm{EX}^{2.2} \mathrm{Box}^{4+}$ \\
\hline Gas Phase & 161 & 171 & 163 & 162 & 279 \\
Toluene & 164 & 172 & 167 & 164 & 171 \\
Chloroform & 164 & 172 & 167 & 163 & 171 \\
Ethanol & 163 & 171 & 166 & 163 & 171 \\
Methanol & 163 & 171 & 166 & 162 & 170 \\
1,2-ethandiol & 163 & 171 & 166 & 163 & 170 \\
Water & 163 & 170 & 166 & 162 & 170 \\
N-Methylformamide-Mixture & 163 & 171 & 166 & 163 & 170 \\
\hline
\end{tabular}

The increasing of the wavelength of main band means the less required energy for electronic transition. According to Table 1, the wavelength of main bands are generally similar to each other in each studied compound except $\mathrm{EX}^{2.2} \mathrm{Box}^{4+}$ for the whole solvents. So, the wavelength was unchanged depending on the solvent. These results affect the optical properties and these properties are similar in each solvent. However, the biggest change occurs in $\mathrm{EX}^{2.2} \mathrm{Box}^{4+}$. The wavelength of main band is calculated as $279 \mathrm{~nm}$ in gas phase and nearly $170 \mathrm{~nm}$ in the other solvent areas. The wavelength of main band is decreased by the increasing of dielectric constant of solvents. Therefore, the optical property of $\mathrm{EX}^{2.2} \mathrm{Box}^{4+}$ decreases in solvents. As a results, $\mathrm{EXBox}^{4+}$ and $\mathrm{EX}^{2.2} \mathrm{Box}^{4+}$ can be used as an optical material in solvent while $\mathrm{EX}^{2.2} \mathrm{Box}^{4+}$ is the best candidate for NLO application in gas phase.

\subsection{Quantum chemical descriptors for NLO properties}

Optical properties can be proposed by using quantum chemical descriptors or parameters. The effect of quantum chemical parameters on the NLO activity has been identified in detail in many published theoretical papers [16-19]. Calculated QCDs in gas phase are given in Table 2 while the other results in toluene, chloroform, ethanol, methanol, 1,2-ethanediol, water and n-methylformamide-mixture are given in Supplemental Material.

Table 2. Calculated quantum chemical descriptors for urea and studied $\operatorname{Ex}^{\mathrm{n}}$ Boxes in gas phase

\begin{tabular}{llllllllll}
\hline Compounds & $\mathrm{E}_{\mathrm{HOMO}}{ }^{\mathrm{a}}$ & $\mathrm{E}_{\mathrm{LUMO}^{\mathrm{a}}}$ & $\mathrm{I}^{\mathrm{a}}$ & $\mathrm{A}^{\mathrm{a}}$ & $\mathrm{E}_{\mathrm{GAP}}{ }^{\mathrm{a}}$ & $\eta^{\mathrm{a}}$ & $\sigma^{\mathrm{b}}$ & $\sigma_{\mathrm{O}}{ }^{\mathrm{b}}$ & $\chi^{\mathrm{a}}$ \\
\hline BlueBox $^{4+}$ & -18.221 & -9.163 & 18.221 & 9.163 & 9.058 & 4.529 & 0.221 & 0.110 & 13.692 \\
EXBox $^{4+}$ & -15.638 & -7.156 & 15.638 & 7.156 & 8.482 & 4.241 & 0.236 & 0.118 & 11.397 \\
EX $^{2}$ Box $^{4+}$ & -5.167 & 3.718 & 5.167 & -3.718 & 8.884 & 4.442 & 0.225 & 0.113 & 0.725 \\
EX $^{0.8}$ Box $^{4+}$ & -4.633 & 2.434 & 4.633 & -2.434 & 7.068 & 3.534 & 0.283 & 0.141 & 1.099 \\
EX $^{2.2}$ Box $^{4+}$ & -4.320 & 1.606 & 4.320 & -1.606 & 5.926 & 2.963 & 0.338 & 0.169 & 1.357 \\
Urea $^{\text {Compounds }}$ & -10.988 & 5.465 & 10.988 & -5.465 & 16.453 & 8.227 & 0.122 & 0.061 & 2.761 \\
\hline BlueBox $^{4+}$ & -13.692 & 20.696 & 0.048 & 3.023 & 0.093 & 45.466 & 114.2771 & $3.70 \times 10^{-28}$ & \\
EXBox $^{4+}$ & -11.397 & 15.314 & 0.065 & 2.687 & 0.000 & 76.696 & 185.8248 & $1.38 \times 10^{-31}$ & \\
EX $^{2}$ Box $^{4+}$ & -0.725 & 0.059 & 16.925 & 0.163 & 0.000 & 50.704 & 111.5649 & $5.46 \times 10^{-32}$ & \\
EX $^{0.8}$ Box $^{4+}$ & -1.099 & 0.171 & 5.847 & 0.311 & 0.000 & 91.900 & 216.5514 & $2.51 \times 10^{-31}$ & \\
EX $^{2.2}$ Box $^{4+}$ & -1.357 & 0.311 & 3.219 & 0.458 & 0.000 & 158.607 & 396.4361 & $1.45 \times 10^{-31}$ & \\
Urea $^{\mathrm{a}}$ & -2.761 & 0.463 & 2.158 & 0.336 & 1.810 & 1.898 & 8.082826 & $3.81 \times 10^{-28}$ & \\
\hline
\end{tabular}

${ }^{\mathrm{a}}$ in $\mathrm{eV}$,

$\mathrm{b}$ in $\mathrm{eV}^{-1}$,

${ }^{c}$ in Debye,

d in $\AA^{3}$,

e $\mathrm{cm}^{5} / \mathrm{esu}$ 
According to Table 4, NLO activity ranking should be as follow for mentioned descriptors:

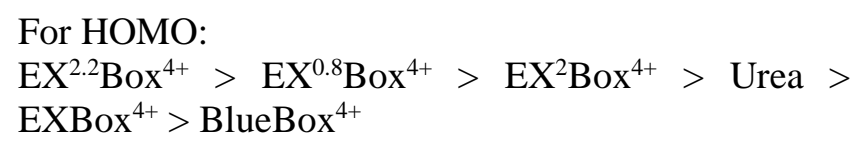

For $\mu$ :

Urea > BlueBox ${ }^{4+}>\mathrm{EX}^{2.2} \mathrm{Box}^{4+}=\mathrm{EX}^{0.8} \mathrm{Box}^{4+}=$ $\mathrm{EX}^{2} \mathrm{Box}^{4+}=\mathrm{Urea}=\mathrm{EXBox}^{4+}$

For $\alpha$ :

$\mathrm{EX}^{2.2} \mathrm{Box}^{4+}>\mathrm{EX}^{0.8} \mathrm{Box}^{4+}>\mathrm{EXBox}^{4+}>\mathrm{EX}^{2} \mathrm{Box}^{4+}>$ BlueBox $^{4+}>$ Urea

For $\Delta \alpha$ :

$\mathrm{EX}^{2.2} \mathrm{Box}^{4+}>\mathrm{EX}^{0.8} \mathrm{Box}^{4+}>\mathrm{EXBox}^{4+}>\mathrm{BlueBox}^{4+}>$ $\mathrm{EX}^{2} \mathrm{Box}^{4+}>$ Urea

For $\beta$ :

Urea > BlueBox ${ }^{4+}>\mathrm{EX}^{0.8} \mathrm{Box}^{4+}>\mathrm{EX}^{2.2} \mathrm{Box}^{4+}>$ $\mathrm{EXBox}^{4+}>\mathrm{EX}^{2} \mathrm{Box}^{4+}$

According to above rankings, the general activity ranking is given as follow:

$\mathrm{EX}^{2.2} \mathrm{Box}^{4+}>\mathrm{EX}^{0.8} \mathrm{Box}^{4+}>\mathrm{EXBox}^{4+}>\mathrm{EX}^{2} \mathrm{Box}^{4+}>$ BlueBox $^{4+}>$ Urea
In gas phase, $\mathrm{EX}^{2.2} \mathrm{Box}^{4+}$ is determined as the best candidate for NLO applications. Additionally, the same analyses are performed in each area and $\mathrm{EX}^{2.2} \mathrm{Box}^{4+}$ is determined as the best material for NLO application, too. As the final word, the results in quantum chemcial parameters are in agreement with contour plots, DOS spectrum, UV-VIS spectrum. It can be said that, $\mathrm{EX}^{2.2} \mathrm{Box}^{4+}$ is the best material respect to all analyzes in this article.

\section{Conclusions}

We presented the computational investigations of some $\mathrm{Ex}^{\mathrm{n}}$ Box Cyclophanes performed at $\mathrm{HF} / 6-31 \mathrm{G}(\mathrm{d})$ level in different solvents. Structural and electronic properties of the mentioned compounds underwent scrutiny in detail. The energy diagram of molecular orbitals and contour plots of significant molecular orbitals and counter plots of significant molecular orbitals are calculated and examined. Additionally, density of state (DOS) spectrum was calculated and according to DOS spectrum, electronic mobility in $\mathrm{EX}^{2.2} \mathrm{Box}^{4+}$ and $\mathrm{EX}^{0.8} \mathrm{Box}^{4+}$ is more than the other. UVVIS spectrum and some quantum chemical descriptors are calculated and $\mathrm{EX}^{2.2} \mathrm{Box}^{4+}$ is found as the best material to NLO applications.

\section{Acknowledgment}

The numerical calculations reported in this paper were fully/partially performed at TUBITAK ULAKBIM, High Performance and Grid Computing Center (TRUBA resources). Also, M. R thanks Professor Sir J. Fraser Stoddarts for giving the opportunity to learn this field of Chemistry during the sabbatical time at Northwestern University in 2016.

\section{Conflicts of interest}

The authors state that did not have conflict of interests

\section{References}

[1] Barnes J.C., Juricek M., Vermeulen N.A., Dale E.J. and Stoddart J.F., Synthesis of $\mathrm{Ex}^{\mathrm{n} B o x}$ Cyclophanes. J. Org. Chem., 78 (2013) 1196211969.

[2] Dale E.J., Vermeulen N.A., Juricek M., Barnes J.C., Young R.M., Wasielewski M.R. and Stoddart J.F., Supramolecular Explorations: Exhibiting the Extent of Extended Cationic Cyclophanes. Acc. Chem. Res., 49 (2016) 262273. 
[3] Dale E.J., Ferris D.P., Vermeulen N.A., Hanklis J.J., Popovs I., Juricek M., Barnes J.C., Schneebeli S.T. and Stoddart J.F., Cooperative Reactivity in an Extended-Viologen-Based Cyclophane. J. Am. Chem. Soc., 138 (2016) 3667-3670.

[4] Fang Q.-S., Chen L. and Liu Q.-L., Templatedirected synthesis of pyridazine-containing tetracationic cyclophane for construction of [2] rotaxane. Chinese Chemical Letters 28 (2017) 1013-1017.

[5] Mashraqui S.H., Sangvikar Y.S., Ghadigaonkar S.G., Ashraf M. and Meetsma M., Oxa-bridged cyclophanes featuring thieno[2,3b]thiopheneand C2-symmetric binol or bisnaphthol rings:synthesis, structures, and conformational studies. Tetrahedron 64 (2008) 8837-8842.

[6] Giancarlo L.C. and Flynn G.W., Raising Flags: Applications of Chemical Marker Groups To Study Self-Assembly, Chirality, and Orientation of Interfacial Films by Scanning Tunneling Microscopy. Acc. Chem. Res. 33 (2000) 491501.

[7] Asakawa M., Ashton P.R., Menzer S., Raymo F.M., Stoddart J.F., White A.J.P. and Williams D.J., Cyclobis(Paraquat-4,4'-Biphenylene)-an Organic Molecular Square. Chem. Eur. J., 2 (1996) 877-893.

[8] Barnes J.C., Juríček M., Strutt N.L., Frasconi M., Sampath S., Giesener M.A., McGrier P.L., Bruns C.J., Stern C.L., Sarjeant A.A. and Stoddart J.F., ExBox: A Polycyclic Aromatic Hydrocarbon Scavenger. J. Am. Chem. Soc., 135 (2013) 183-192.

[9] Juríček M., Barnes J.C., Dale E.J., Liu W.-G., Strutt N.L., Bruns C.J., Vermeulen N.A., Ghooray K., Sarjeant A.A., Stern C.L., Botros Y.Y., Goddard W.A. III and Stoddart J.F., Ex2Box: Interdependent Modes of Binding in a Two-Nanometer-Long Synthetic Receptor. $J$. Am. Chem. Soc., 135 (2013) 12736-12746.

[10] Asakawa M., Dehaen W., L'abbe G.L., Menzer S., Nouwen J., Raymo F. M., Stoddart J. F. and Williams D.J., Omproved Template-Directed Synthesis of Cyclobis(paraquat-p-phenylene). $J$. Org. Chem., 61 (1996) 9591-9595.

[11] Dennington II R.D., Keith T.A. and Millam J.M., Gauss View 5.0, Wallingford, CT (2009).

[12] Gaussian 09, Revision D.01, Frisch M. J., Trucks G. W., Schlegel H. B., Scuseria G. E., Robb M. A., Cheeseman J. R., Scalmani G., Barone V., Petersson G. A., Nakatsuji H., Li X.,
Caricato M., Marenich A. V., Bloino J., Janesko B.G., Gomperts R., Mennucci B., Hratchian H. P., Ortiz J. V., Izmaylov A. F., Sonnenberg J. L., Williams-Young D., Ding F., Lipparini F., Egidi F., Goings J., Peng B., Petrone A., Henderson T., Ranasinghe D., Zakrzewski V. G., Gao J., Rega N., Zheng G., Liang W., Hada M., Ehara M., Toyota K., Fukuda R., Hasegawa J., Ishida M., Nakajima T., Honda Y., Kitao O., Nakai H., Vreven T., Throssell K., Montgomery J.A. Jr., Peralta J. E., Ogliaro F., Bearpark M.J., Heyd J.J., Brothers E.N., Kudin K.N., Starovero, V.N., Keith T.A., Kobayashi R., Normand J., Raghavachari K., Rendell A.P., Burant J.C., Iyengar S.S., Tomasi J., Cossi M., Millam J.M., Klene M., Adamo C., Cammi R., Ochterski J.W., Martin R.L., Morokuma K., Farkas O., Foresman J. B. and Fox D. J. Gaussian, Inc., Wallingford CT, (2009).

[13] PerkinElmer, ChemBioDraw Ultra Version (13.0.0.3015), CambridgeSoft Waltham, MA, USA (2012).

[14] O'Boyle N.M., Tenderholt A.L. and Langner K.M.: cclib: A library for package-independent computational chemistry algorithms. J Comp Chem, 29 (2008) 839-845.

[15] Koopmans T., Über die Zuordnung von Wellenfunktionen und Eigenwerten zu den Einzelnen Elektronen Eines Atoms. Physica, 1 (1934) 104-113.

[16] Tüzün B. and Sayin K., Investigations over optical properties of boron complexes of benzothiazolines. Spectrochim. Acta A, 208 (2019) 48-46.

[17] Uludag N. and Serdaroğlu G., An improved synthesis, spectroscopic (FT-IR, NMR) study and DFT computational analysis (IR, NMR, UVeVis, MEP diagrams, NBO, NLO, FMO) of the 1,5-methanoazocino[4,3-b]indole core structure. J. Mol. Struct., 1155 (2018) 548-560.

[18] Sayin K., Kariper S.E., Tas M., Alagoz Sayin T. and Karakas D., Investigations of structural, spectral, electronic and biological properties of $\mathrm{N}$-heterocyclic carbene $\mathrm{Ag}(\mathrm{I})$ and $\mathrm{Pd}(\mathrm{II})$ complexes. J. Mol. Struct., 1176 (2019) 478487.

[19] Gungor S.A., Sahin I., Gungor O., Kariper S.E., Tumer F. and Kose M., Pamoic acid esters and their xanthene derivatives: Flourimetric detection of nitroaromatic compounds and nonlinear optical properties. Sensors and Actuators B: Chemical, 255 (2018) 3344-3354. 